\title{
RÉSULTATS DE DÉVERSEMENTS, DANS LE LÉMAN, D'OMBLES MARQUÉS
}

\author{
P.J. LAURENT * \\ Avec la collaboration technique de \\ J. ESCOMEL *, Ph. LAURENT * et C. MERCIER **
}

\section{RÉSUMÉ}

Des ombles chevalier de 1 à 2 hivers ont été marqués et lâchés dans le Léman pour voir le devenir de ces poissons de pisciculture dans le lac. Les marques dorsales donnent de meilleurs pourcentages de retours que les marques maxillaires, tandis que le cryomarquage passe inaperçu des pêcheurs une centaine de jours après l'immersion des poissons dans le lac.

La croissance en longueur des poissons d'un hiver est comparable à celle des ombles de pisciculture; à cet âge, dans le lac, le zooplancton constitue la base de la nourriture. Les ombles de deux hivers accusent par contre un retard de croissance important par rapport à ceux du même âge gardés en pisciculture. Le mode de marquage maxillaire pourrait expliquer ce fait bien que sur des ombles d'un hiver les tests statistiques ne révèlent aucune influence du type de marquage sur la croissance en longueur. L'appauvrissement de la faune benthique, proie habituelle des ombles, lié à l'altération de la qualité de l'eau du Léman, pourrait également expliquer ce retard de croissance.

On note chez les ombles marqués une inclination très nette à vivre en bancs et, pour ceux d'entre eux qui sont lâchés le long des côtes sud du lac, une tendance à migrer sur les rives septentrionales. Ce comportement profite aux pêcheurs suisses. Les lâchers d'ombles marqués bénéficient également davantage aux pêcheurs professionnels qu'aux pêcheurs amateurs.

Quelques conclusions sur les meilleures conditions de lâchers d'ombles de pisciculture peuvent être tirées de ce travail qui demande à être complété, après mise au point d'un mode de marquage correct, par une appréciation de l'efficacité des peuplements réalisés avec des alevins.

\section{SUMMARY}

Chars produced in experimental fishfarms have been tagged and released in the lake of Geneva at the age of one and two winters.

Dorsal tags (Carlin's type) give the best percentage of return compared to jaw tags. Cold branding by liquid nitrogen gives poor results : fishermen do not recognize marked fish after 100 days.

Released chars find enough zooplancton in the eutrophised lake of Geneva to sustain a growth comparable to that of fishfarm chars. On the contrary, the length growth of two winters chars released in the lake is slower than the growth of same age fishes fed in the fishfarm. Statistical tests do not prove any influence of tagging on the length growth. But deep bottom fauna impoverishment connected to water quality degradation could be suspected to be responsible for this difference.

Tagged chars show a tendency to live in a shoal and for those released along the French south side to migrate to the Swiss north side of the lake. Most of the tagged chars are caught in the Swiss part of the lake and more frequently by commercial fishermen than by sport ones. Some conclusions can be set up concerning release of one or two winters old firshfarm chars. The value of restocking with less expensive juvenile chars must be evaluated by an appropiate tagging method.

\footnotetext{
* I.N.R.A., Station d'Hydrobiologie Lacustre, 75, Avenue de Corzent, 74203 THONON LES BAINS

* Délégation Régionale du Conseil Supérieur de la Pêche, 285, rue du Quatre Août, 69100 VILLEURBANNE.
} 


\section{INTRODUCTION}

Le Léman reçoit depuis longtemps des contingents importants d'ombles chevalier élevés en pisciculture. On espère par cette pratique contribuer au maintien d'un peuplement précieux dont le déclin n'est malheureusement que trop flagrant depuis une vingtaine d'années. Quel est le devenir des ces ombles? Leur marquage permet de répondre à cette question, du moins lorsqu'il est réalisé sur des ombles de plus de 10 centimètres.

II n'est pas possible par contre de marquer pour l'instant de manière durable, évidente et inoffensive. les très nombreux alevins déversés chaque année et obtenus: soit à partir d'œufs d'ombles sauvages du Léman, pêchés sous contrôle de l'Administration sur des frayères situées à des endroits précis par 60 à 100 mètres de fond, soit à partir d'œufs importés de Scandinavie et récoltés en abondance sur les cours d'eau lors des migrations reproductrices que les poissons de cette région entreprennent depuis la mer ou les lacs.

Sur des poissons d'une taille supérieure à une dizaine de centimètres, des marquages efficaces ont pu être effectués entre 1961 et 1966 avec des estivaux achetés à l'étranger ou produits à la pisciculture domaniale de Thonon. Ultérieurement, la domestication de l'omble chevalier du Léman Salvelinus alpinus (L.) jamais réalisée ailleurs, et entreprise avec succès par la pisciculture expérimentale de l'INRA (Institut National de la Recherche Agronomique), a permis, à la demande du Ministère de l'Environnement, Service de la Pêche et de l'Hydrobiologie, d'apprécier les performances de poissons de plus grande taille.

Au total depuis 1961, 7465 poissons (6370 ombles et 1095 hybrides) ont été ainsi marqués et à partir des résultats obtenus, quelques enseignements peuvent être tirés sur les marquages et les déverisements d'ombles dans le Léman.

\section{Divers types de marques utilisées.}

Trois types de marquages ont été mis en cuvre. Des marques dorsales de notre fabrication analogues aux marques de type Carlin ont été utilisées avec grand succès sur des poissons à forte croissance tels que la truite Arc-en-Ciel Salmo gairdneri Richardson. Appliquées à des ombles de 12 à 13 centimètres de longueur totale, ces marques ont permis d'enregistrer le plus fort pourcentage de retour : $13.0 \%$ (Tableau 1). Cependant, plus de la moitié des poissons marqués sont repris dans le mois qui suit leur déversement, avant qu'ils aient eu le temps de s'accoutumer à la vie en liberté et d'accuser une croissance intéressante (Fig. 1). D'autre part, il a été remarqué sur 4,5\% des ombles repris et contrairement à la truite, qu'une infection du tissu musculaire se produisait au contact du fil inoxydable à usage chirurgical utilisé pour fixer la marque.

Des marques maxillaires Presadom (*), de taille appropriée à la dimension des poissons, ont été également employées. Le pourcentage des retours est très inférieur à celui fourni par les marques dorsales (Tableau 1), mais la répartition des captures dans le temps est plus échelonnée (Fig. 1) et la durée du marquage excellente puisqu'elle atteint presque trois ans. Le marquage maxillaire semble présenter l'inconvénient d'affecter la capacité de préhension des ombles et de vouer les poissons qui portent une marque à une maigreur anormale comparée à celle des ombles non marqués.

Tableau 1 : Comparaison des résultats fournis par les trois types de marquage utilisés.

\begin{tabular}{|c|c|c|c|c|c|}
\hline $\begin{array}{l}\text { Type de } \\
\text { trarques }\end{array}$ & $\begin{array}{c}\text { Nombre ounoles } \\
\text { maqués }\end{array}$ & $\begin{array}{l}\text { Somore de pois- } \\
\text { sons repris }\end{array}$ & $\begin{array}{l}\text { Pourcentage de } \\
\text { retaur }\end{array}$ & $\begin{array}{c}\text { Ouree maximum } \\
\text { de séjour au lac } \\
\text { (jours) }\end{array}$ & Ooservations \\
\hline $\begin{array}{l}\text { Dorsale } \\
\text { (type Carlin) }\end{array}$ & 1183 & 154 & 13.0 & 688 & $\begin{array}{l}\text { Infection musculaire signalee } \\
\text { chez orbles marques }\end{array}$ \\
\hline $\begin{array}{l}\text { Maxillaire } \\
\text { (Presadom) }\end{array}$ & 1587 & 71 & 4.4 & 1062 & $\begin{array}{l}\text { Gene probable au ban fonctionne- } \\
\text { ment des ndicnnires. }\end{array}$ \\
\hline $\begin{array}{l}\text { Gryomorquage } \\
\text { ( } x \text { du cote gauche) }\end{array}$ & $(837)$ & $(20)$ & 2.3 & 103 & $\begin{array}{l}\text { Ombles egalement marques par marque } \\
\text { maxiliaire. }\end{array}$ \\
\hline $\begin{array}{l}\text { Cryomarquage } \\
\text { (1 du cóté droit) }\end{array}$ & 3609 & 0 & 0 & 0 & \\
\hline
\end{tabular}

(*) Square de la Libération, 19100 BRIVES. 
Figure 1 : Pourcentages de retour en fonction du temps des ombles marqués par marques maxillaires et dorsales.

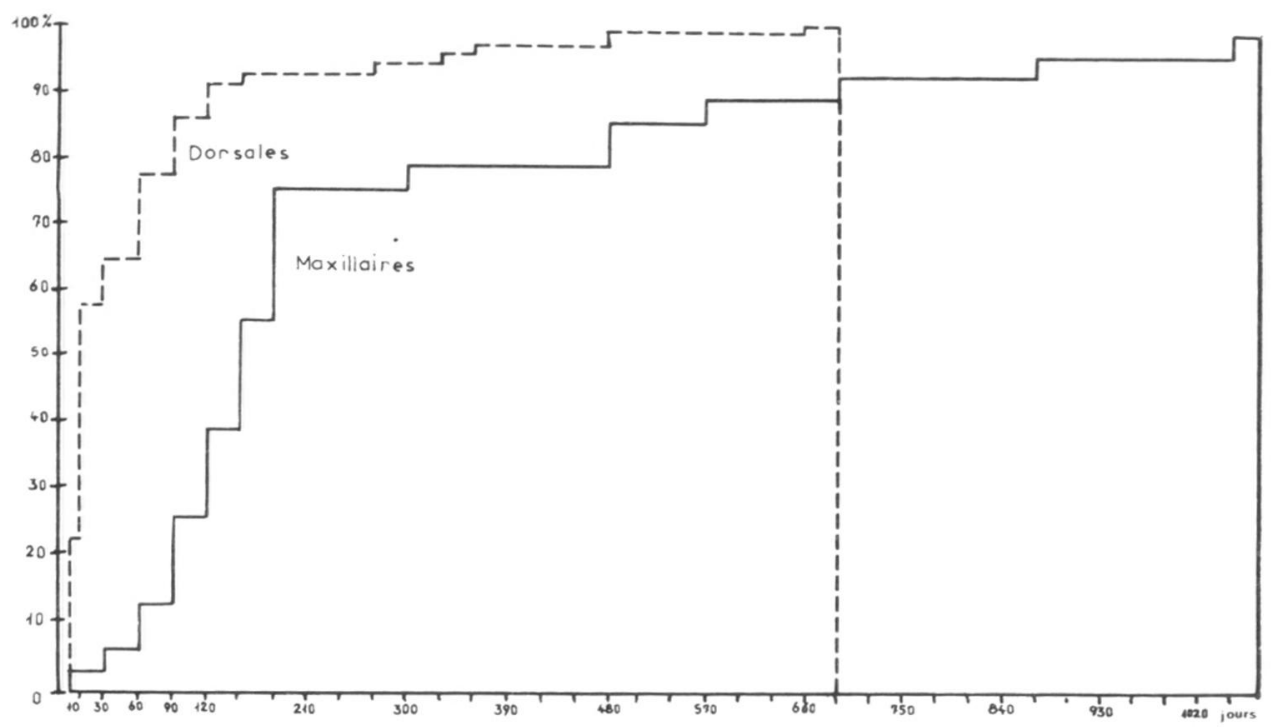

Le cryomarquage, utilisé avec succès sur des espèces fragiles et migratrices telles que le saumon (1), semblait devoir être une méthode de choix pour le repérage de lots d'ombles, y compris des alevins. Des ombles adultes ont reçu un double marquage: maxillaire et cryomarquage à titre d'essai. Les pêcheurs n'ont reconnu le signe de cryomarquage: un $X$ sur le flan gauche (photo 1) que durant les 3 premiers mois des recaptures et sur la moitié du contingent d'ombles repris.

\section{Photo $n^{\circ} 1$ : Omble cryomarqué repris au Léman un mois après sa libération et photographié par le pêcheur qui l'avait capturé (photo Monbaron à Bursinel, Suisse).}

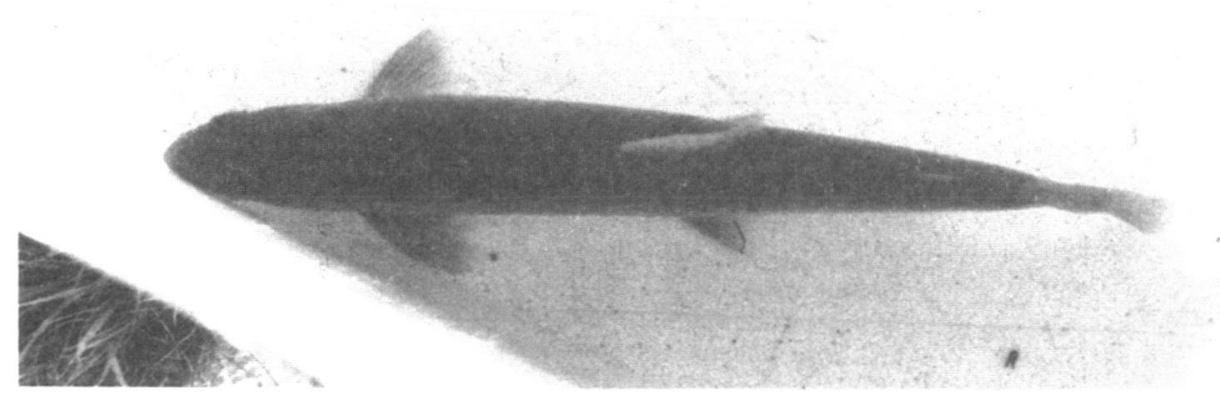

En février 1980, 3600 ombles d'un hiver et d'une longueur totale moyenne de 8,3 centimètres ont également reçu un cryomarquage sous la forme d'un I apposé au flanc droit. Aucun de ces poissons, dont par ailleurs la nageoire adipeuse avait été coupée, n'a pu jusqu'à présent être repéré soit par les pêcheurs, soit lors des pêches exceptionnelles sur les zones de frayères (Tableau 1). Des témoins gardés en pisciculture n'ont permis de retrouver, avec plus ou moins de peine et 300 jours après, que $12 \%$ des sujets encore marqués (photo 2) de la lettre I.

Il faut donc conclure qu'aucun mode de marquage n'est actuellement totalement satisfaisant: les marques dorsales sont inapplicables sur les poissons d'une taille inférieure à 10 centimètres; elles déterminent quelques réactions nécrotiques des tissus de l'omble et elles accroissent la vulnérabilité des poissons aux filets, notamment lorsque les sujets sont encore petits. Les marques maxillaires s'appliquent également mal sur les petits ombles; de plus elles peuvent perturber le fonctionnement normal des mâchoires et entraver la nutrition des poissons. Le cryomarquage s'applique vite, facilement et sans perturbation apparente des ombles, même s'ils sont petits, mais il a 


\section{Photon 2:Omble cryomarqué gardé en pisciculture et photographié 300 jours après son marquage. On distingue au bout de la flèche une barre oblique par rapport à la ligne latérale.}

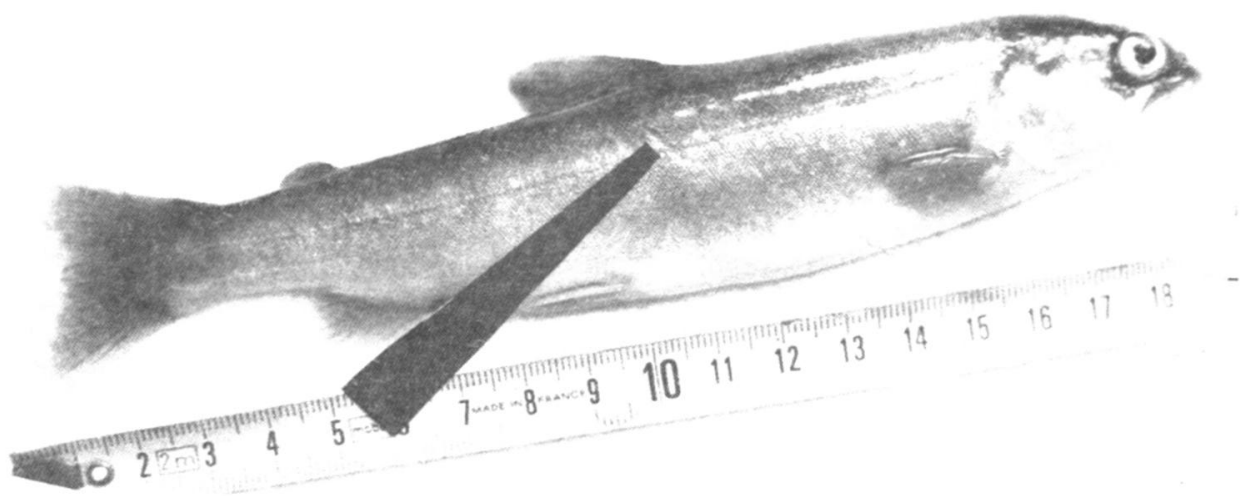

une durée limitée puisque son observation échappe aux pêcheurs après une centaine de jours.

De nouvelles investigations seront nécessaires; elles s'orienteront sans doute vers des marques internes, repérables avec précision aux points de collecte des poissons: grossistes et coopérative. La simple analyse des captures en fonction des lâchers d'alevins non marqués conduirait en effet à des résultats très incertains en raison de l'imprécision des statistiques basées uniquement sur les déclarations des seuls pêcheurs professionnels.

\section{Validité des informations fournies.}

Le succès des campagnes de marquages repose actuellement sur la bonne volonté et la rigueur de ceux qui capturent des ombles marqués. Des questionnaires fournis aux pêcheurs facilitent la collecte des informations et une récompense pour retour de marques et fourniture de renseignements tente d'encourager les bonnes volontés, tant en Suisse qu'en France, puisque les ressources piscicoles du Léman sont exploitées par ces deux pays.

En dépit de ces dispositions, les informations fournies ne sont pas toujours complètes et fiables (Tableau 2); de plus, bien des pêcheurs ne prennent pas la peine de signaler les reprises de poissons marqués.

\section{Tableau 2 : Validité des renseignements fournis en pourcentage du nombre total des réponses.}

\begin{tabular}{|c|c|c|c|}
\hline $\begin{array}{l}\text { Renseignements } \\
\text { complets et valides }\end{array}$ & $\begin{array}{c}\text { Renseignements } \\
\text { incomplets mais exacts }\end{array}$ & $\begin{array}{c}\text { Renseignements } \\
\text { complets inexacts }\end{array}$ & $\begin{array}{c}\text { Absence de } \\
\text { renseignements }\end{array}$ \\
\hline $45.6 \%$ & $25.6 \%$ & $10.2 \%$ & $17.6 \%$ \\
\hline
\end{tabular}

Moins de la moitié des réponses sont complètes et plausibles et plus d'un dixième des renseignements fournis sont inexacts. Ces inexactitudes sont flagrantes lorsqu'il s'agit de la longueur totale des poissons dont certains sujets se retrouvent plus petits quelque temps après le marquage qu'avant. Mais les erreurs les plus communes concernent le poids des poissons dont les valeurs sont toujours incertaines et semblent résulter davantage d'évaluations que de pesées rigoureuses.

L'ensemble de ces constatations s'applique certainement à n'importe quelle campagne de marquage, mais il nous a semblé intéressant d'indiquer dans le cas du Léman le degré de validité des renseignements fournis par les pêcheurs.

\section{Croissance des ombles marqués.}

Elle n'a pu être estimée que sur la longueur totale déclarée par les pêcheurs à la suite d'une capture d'omble marqué. Toutefois dans les premières semaines après le 
déversement, il ne faut pas s'attendre à un allongement appréciable des poissons et si l'on porte attention à leur poids, on s'aperçoit, en dépit des incertitudes liées à la mesure de ce paramètre par les pêcheurs, qu'il y a un amaigrissement des ombles nouvellement déversés.

Dans le mois qui suit leur déversement, les ombles de 13 centimètres de longueur totale perdent $4,1 \mathrm{~g} \pm 8,7 \mathrm{~g}$, à $95 \%$ de limite de confiance, et dans le même temps des ombles adultes de 23,8 centimètres de longueur totale, $13,6 \mathrm{~g} \pm 19,8 \mathrm{~g}$ au même seuil de confiance.

Après marquage, l'accroissement en longueur d'ombles d'un hiver et de 13 centimètres de longueur totale moyenne n'est pas extrêmement rapide (Fig. 2) mais il est cependant meilleur que celui des ombles du lac de Zoug (6). Il est intéressant de comparer cette croissance à celle d'ombles de pisciculture de même taille : les allongements dans le lac ou en bassin sont comparables.

\section{Figure 2 : Croissance en longueur d'ombles marqués à la taille moyenne de 13 centimètres de longueur totale. Les traits verticaux indiquent les variations de longueur à la limite de confiance de $95 \%$ pour les groupes d'ombles pêchés après des temps de séjour au lac identiques * ou de lots de poissons de même origine mais gardés en pisciculture - Les signes 0 indiquent les croissances de poissons repris isolément.}

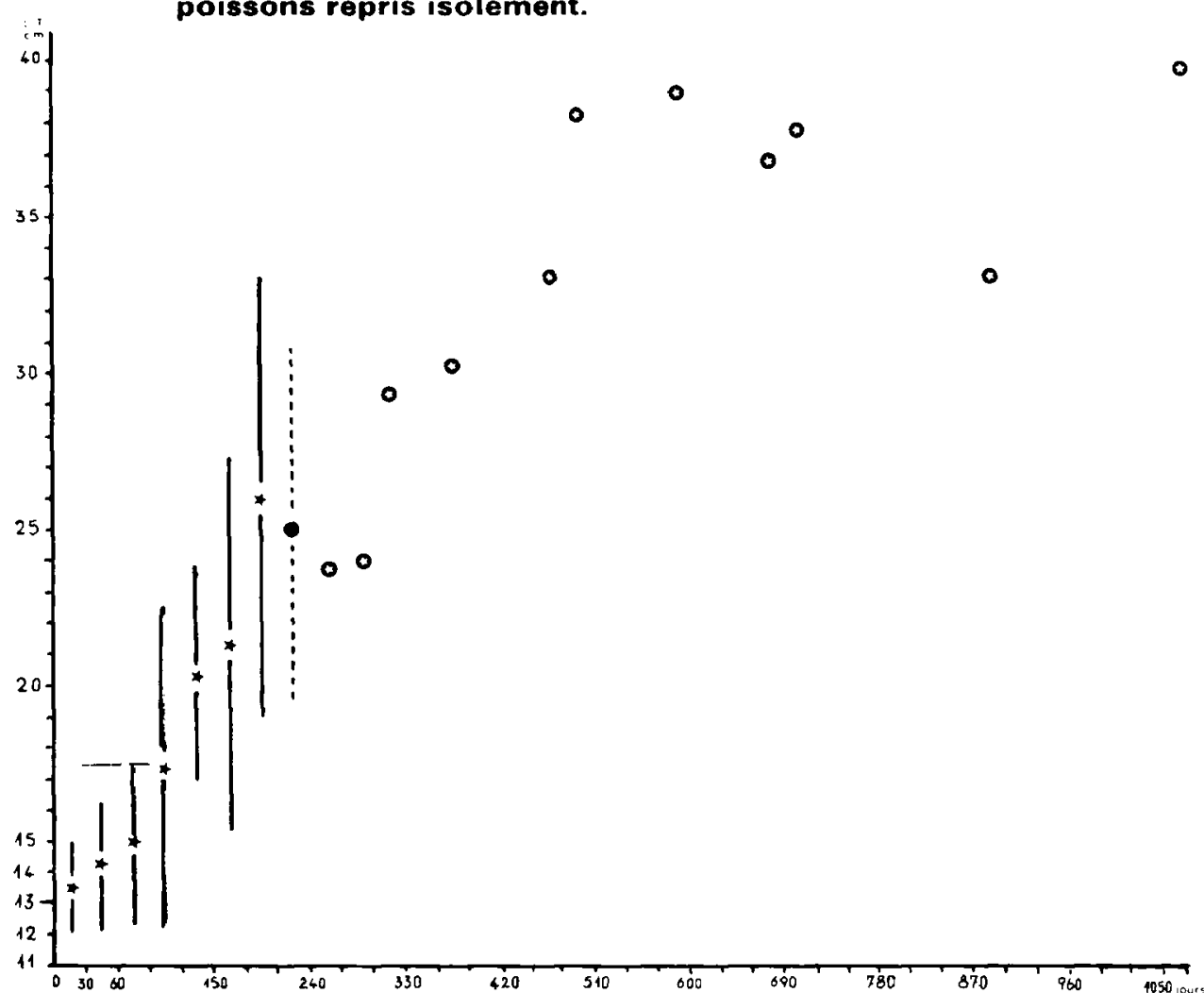

Chez les ombles adultes de deux hivers et de 23,8 centimètres de longueur totale moyenne, déversés en octobre, l'allongement est encore moins rapide que chez les jeunes (Fig. 3): d'autre part, cette croissance, comparée à celle obtenue dans le même temps en pisciculture sur des poissons de même taille et de même âge, nourris aux granulés, est beaucoup moins rapide.

Le mode de marquage, dorsal ou maxillaire, influe-t-il sur la croissance en longueur des poissons marqués? La réponse à cette question peut être trouvée en examinant les résultats obtenus sur un lot de 1500 ombles de 13 centimètres achetés en Suède et déversés en mars 1966 après marquage de la moitié de ce contingent par les deux types de marques. 
Figure 3 : Croissance en longueur d'ombles marqués à la taille moyenne de 23,8 centimètres de longueur totale. Même signification des signes que sur la figure 2.

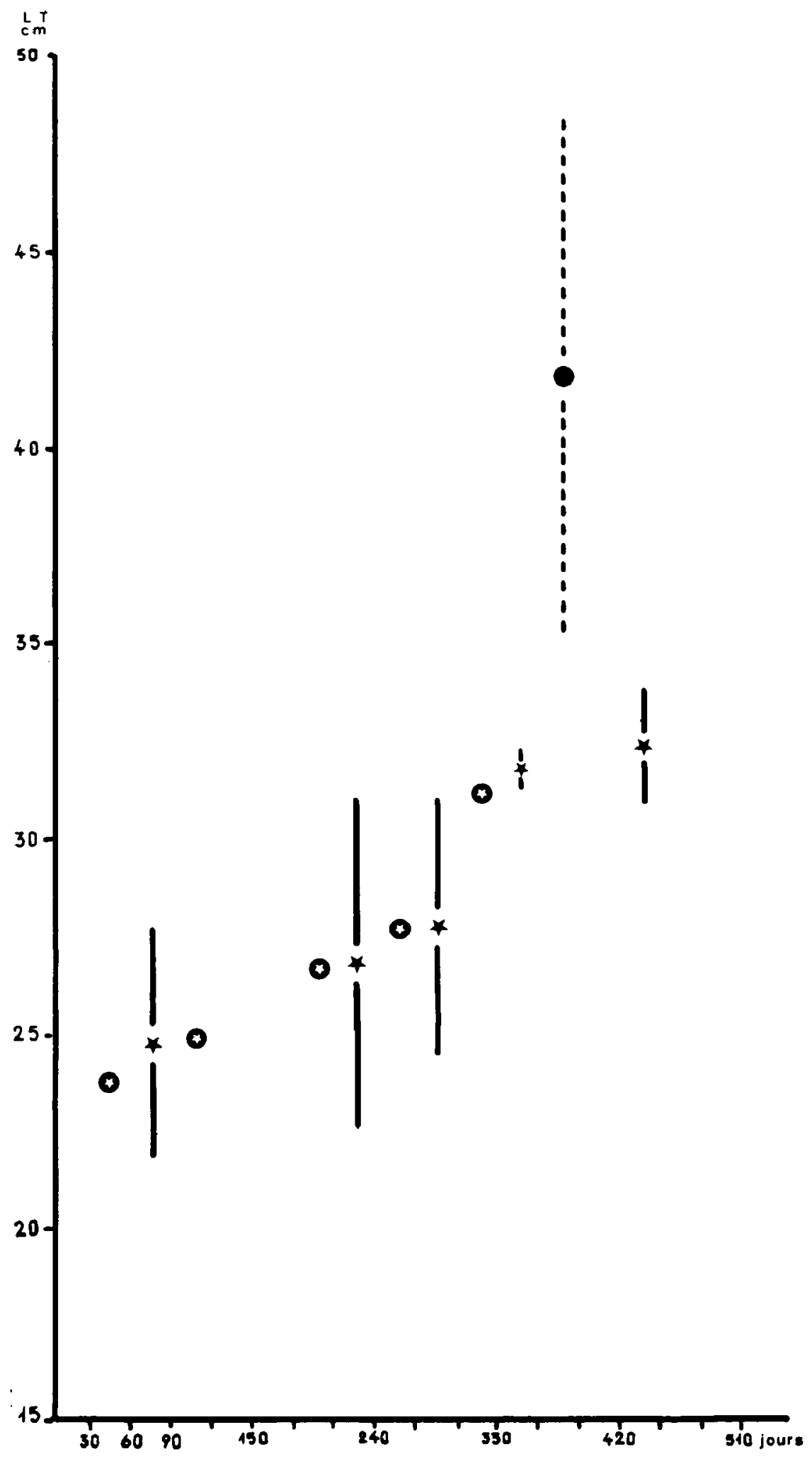


On compare la croissance journalière, multipliée par 100 , des poissons des deux lots qui ont été repris et sur lesquels on dispose de données. Le calcul de la variable de Snedecor (test de F) et la comparaison des moyennes obtenues (loi de Student Fisher) pour les deux lots d'ombles, conduisent au même résultat : avec l'échantillon limité qui a été étudié il n'a pas été mis en évidence d'inflluence significative du mode de marquage sur l'allongement des poissons (Tableau 3).

\section{Tableau 3 : Comparaison après capture de l'allongement journalier exprimé sous la forme:

$$
\begin{aligned}
& \text { ailongement constaté : } \Delta \frac{1}{\text { temps de séjour au lac en jours : } T}+100
\end{aligned}
$$ \\ pour des ombles de 13 centimètres de longueur totale marqués par marques maxillaires et dorsales et déversés en mars 1966.}

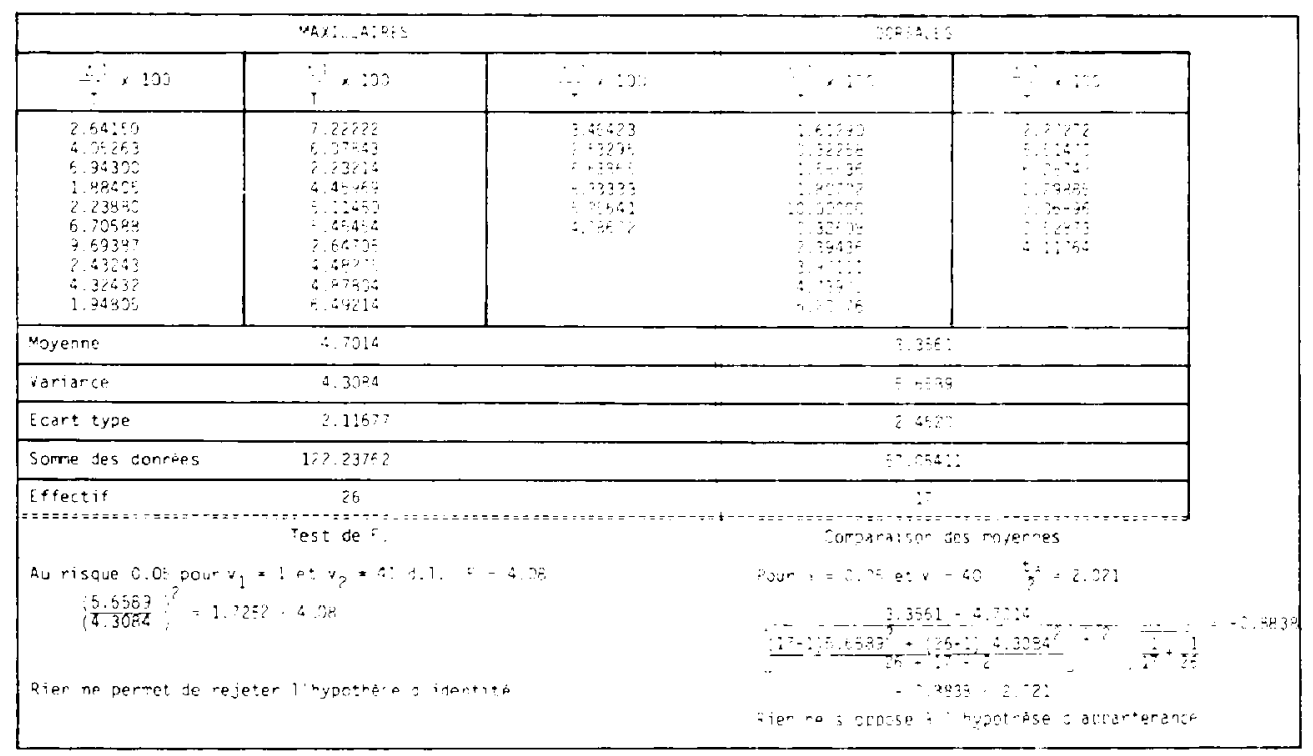

Des résultats différents auraient sans doute été obtenus s il avait été possible de disposer de données fiables sur la croissance pondérale des ombles. Les quelques poissons porteurs de marques maxillaires que nous avons pu contrôler étaient tous anormalement maigres. On peut citer les deux ombles repris sur l'omblière de Meillerie en décembre 1981 : I'un, une femelle de $29,5 \mathrm{~cm}$, pesait $165 \mathrm{~g}$ et l'autre, un mâle de $28,9 \mathrm{~cm}, 170 \mathrm{~g}$, alors qu'en se référant à la relation tongueur/poids d'ombles sauvages et non marqués de même taille, ils auraient dû respectivement peser 294 et $242 \mathrm{~g}$.

\section{Quelques points de la biologie de l'omble révélés par le marquage.}

Les retours de poissons marqués montrent que les ombles restent groupés (Tableau 4). S'il n'y a rien d'étonnant à ce que des poissons marqués se fassent reprendre ensemble sur les lieux même de leur lâcher, peu de jours après leur déversement, il est remarquable par contre que le même pêcheur puisse reprendre dans le même filet, plusieurs mois après leur libération et loin de leur point de déversement au même endroit, des poissons issus du même lot. Bien entendu, les marques dorsales, qui s'accrochent dans les filets même si le poisson est trop petit pour être retenu par la maille employée, montrent beaucoup mieux la grégarité des ombles que les marques maxillaires.

On remarque également que le même pêcheur capture dans la même zone et à quelques jours d'intervalle des ombles marqués issus du même déversement. Incontestablement l'omble est un animal qui vit en bancs et ce fait n'a pas échappé aux pêcheurs, tant à ceux qui pêchent à la ligne traînante qu'à ceux qui les capturent au filet. Les travaux de FROST avaient d'ailleurs montré que l'alevin d'omble anglais est un poisson grégaire mieux armé par ce comportement pour la vie pélagique et l'exploitation du plancton (2). Ceux de HOGLUND et ASTRAND ont prouvé d'autre part la nature olfactive de ce comportement chez de jeunes Salvelinus alpinus (3). 
Tableau 4 : Recaptures d'ombles marqués et déversés ensembles.

\begin{tabular}{|c|c|c|c|c|c|}
\hline $\begin{array}{l}\text { Duree de sejour } \\
\text { au lac } \\
\text { en jours }\end{array}$ & $\begin{array}{l}\text { Lieu de } \\
\text { jêversement }\end{array}$ & $\begin{array}{l}\text { Lieu de } \\
\text { recapture }\end{array}$ & $\begin{array}{c}\text { vombre de poissons } \\
\text { rarqués et déversés } \\
\text { ensemble pus resris } \\
\text { ensernole }\end{array}$ & $\begin{array}{c}\text { Taille moyenne } \\
\text { des poissans. } \\
\text { longueur totale } \\
\text { en cm } \\
\text { au déversement }\end{array}$ & Type de marques \\
\hline : & Excenevex & Excenevex & 15 & 13 & gorsales \\
\hline 3 & rvoire & Messery & $\hat{\imath}$ & 13 & dorsales \\
\hline 3 & 5: $=$ rex & St $=\operatorname{rex}$ & 4 & 13 & dorsales \\
\hline 3 & Thonon-Corzent & 201:e & 2 & 23.8 & maxillaires \\
\hline 3 & Plage Thonor. & zipaije & 2 & 13 & dorsales \\
\hline 11 & P:age :nanon & Pipaille & 2 & 13 & Jorsales \\
\hline 14 & St Prex & St Prex & 2 & 13 & dorsales \\
\hline 21 & $D$ agge Thonon & Milieu du lac & $?$ & $: 3$ & dorsales \\
\hline 32 & Fnonon Corzent & Montreux & 2 & 23.3 & maxifidires \\
\hline 9.3 & vroire & Zyon & $\hat{\imath}$ & $: 3$ & dorsales \\
\hline 87 & St Prex & Roile & 2 & 13 & gorsales \\
\hline
\end{tabular}

Il est également intéressant de noter que la plupart des ombles déversés sur la côte française se déplacent très rapidement en direction des côtes suisses et notamment vers la région de Nyon et Rolle. Ainsi sur un lot de 837 ombles de deux hivers, d'une longueur moyenne de 23,8 centimètres déversés au bord du lac, devant la pisciculture de I'INRA à Thonon-Corzent, tous les poissons (1 7 individus) repris dans les trente jours qui ont suivi ce lâcher ont été capturés sur les côtes suisses (Fig. 4). 24 heures suffisent parfois pour que le poisson se déplace de plus de $18 \mathrm{~km}$.

Figure 4 : Déplacements d'ombles d'une taille moyenne de 23,8 centimètres dans le mois qui suit leur déversement à côté de Thonon. Les chiffres notés à côté des trajets accomplis indiquent le nombre de poissons repris dans la même zone et durant la même période

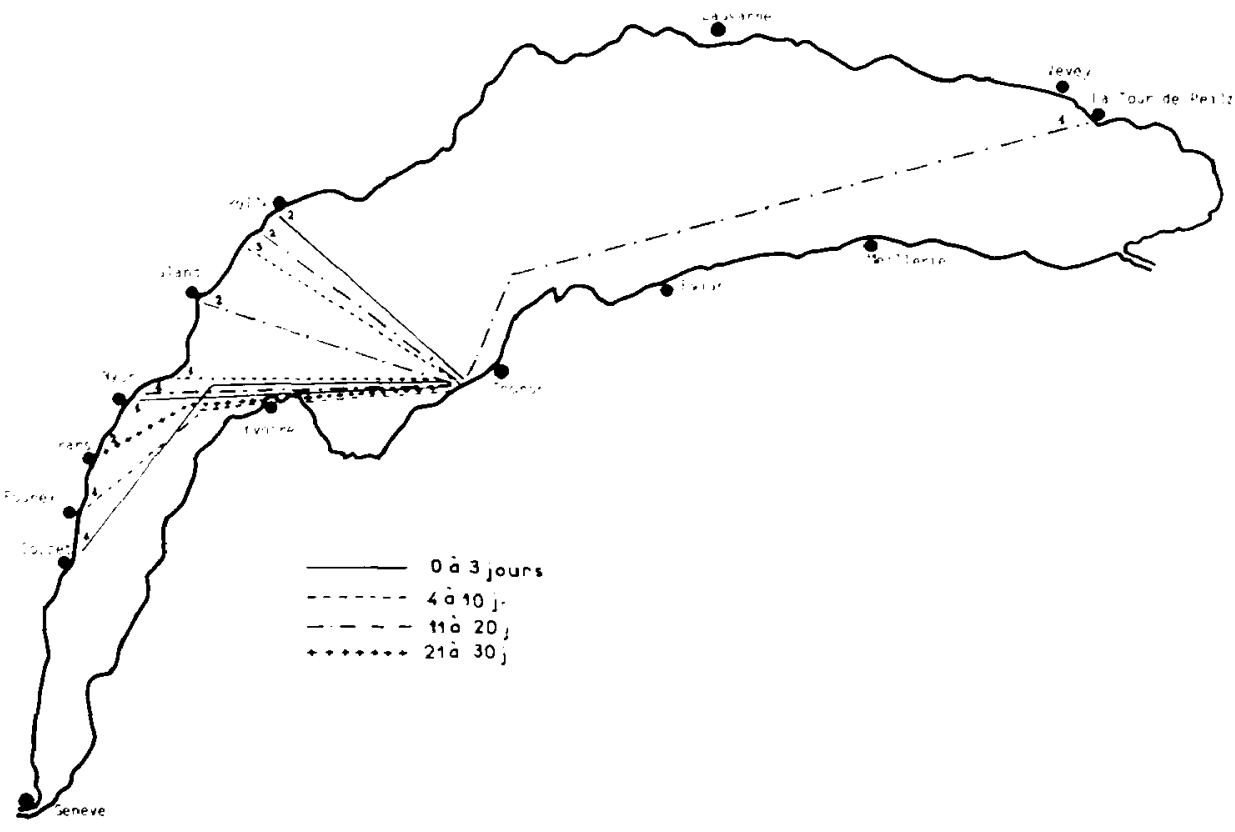


Cette tendance se retrouve sur tous les lots d'ombles et alors que $91 \%$ des animaux marqués ont été déversés le long des côtes françaises, plus de $61 \%$ de ceuxqui ont été recapturés ont été pris le long des côtes suisses (Tableau 5 et Fig. 5). Les lâchers sont toujours plus favorables à la Suisse qu'à la France; seuls des déversements effectués à l'embouchure de la Dranse et à Meillerie (zones 3 et 4 ) donnent un léger avantage aux pêcheurs français.

Tableau 5 : Migrations observables lors des recaptures.

\begin{tabular}{|c|c|c|c|c|c|c|c|c|c|c|c|c|}
\hline \multicolumn{3}{|c|}{ Jeversements } & \multicolumn{7}{|c|}{ Recapture en nomore de po'ssors } & \multicolumn{2}{|c|}{-ocaux } & \\
\hline & & \multirow{2}{*}{$\begin{array}{l}\text { nonbre } \\
\text { ceversé }\end{array}$} & Zone: & \multicolumn{2}{|c|}{ Zore 2} & \multicolumn{2}{|c|}{ Zone 3} & \multicolumn{2}{|c|}{ Zone 4} & \multirow[b]{2}{*}{ Suisse } & \multirow[b]{2}{*}{ Frarce } & \\
\hline & & & Suisse & Suise & France & suisse & France & S-isse & =rance & & & \\
\hline zone: & suisse & 了 & - & - & - & & - & - & - & - & - & \\
\hline \multirow{2}{*}{ 2ore 2} & Suisse & J & - & - & $\cdot$ & - & - & $\cdot$ & - & - & - & \\
\hline & France & 1232 & $: 8$ & 43 & 33 & $\vdots$ & 2 & 5 & : & 68 & 36 & \\
\hline \multirow{2}{*}{ 2ore 3} & Suisse & 253 & : & s & : & is & 2 & 0 & 1 & 21 & 4 & \\
\hline & France & 983 & 3 & $: 4$ & 5 & $s$ & $: 9$ & 3 & $z$ & 28 & 31 & \\
\hline \multirow{2}{*}{ Zone 4} & Suisse & 0 & - & - & - & - & - & - & - & - & - & \multirow{2}{*}{$\begin{array}{l}\text { Yotai } \\
\text { des } \\
\text { reprises }\end{array}$} \\
\hline & France & $3=0$ & 3 & 8 & 2 & 6 & 4 & : & 7 & $: 8$ & 13 & \\
\hline \multirow{2}{*}{ Tctal $x$} & Suisse & 250 & \multirow{2}{*}{25} & \multirow{2}{*}{3} & \multirow{2}{*}{$\because:$} & \multirow{2}{*}{$\therefore$} & \multirow{2}{*}{26} & \multirow{2}{*}{9} & \multirow{2}{*}{$: 7$} & \multirow{2}{*}{335} & \multirow{2}{*}{84} & \multirow{2}{*}{210} \\
\hline & irance & 2520 & & & & & & & & & & \\
\hline
\end{tabular}

Figure 5 : Découpage du lac Léman en zones montrant les points de déversement et les lieux de recapture des ombles marqués. En pointillé la frontière entre la Suisse et la France. Ce croquis illustre le tableau 5.

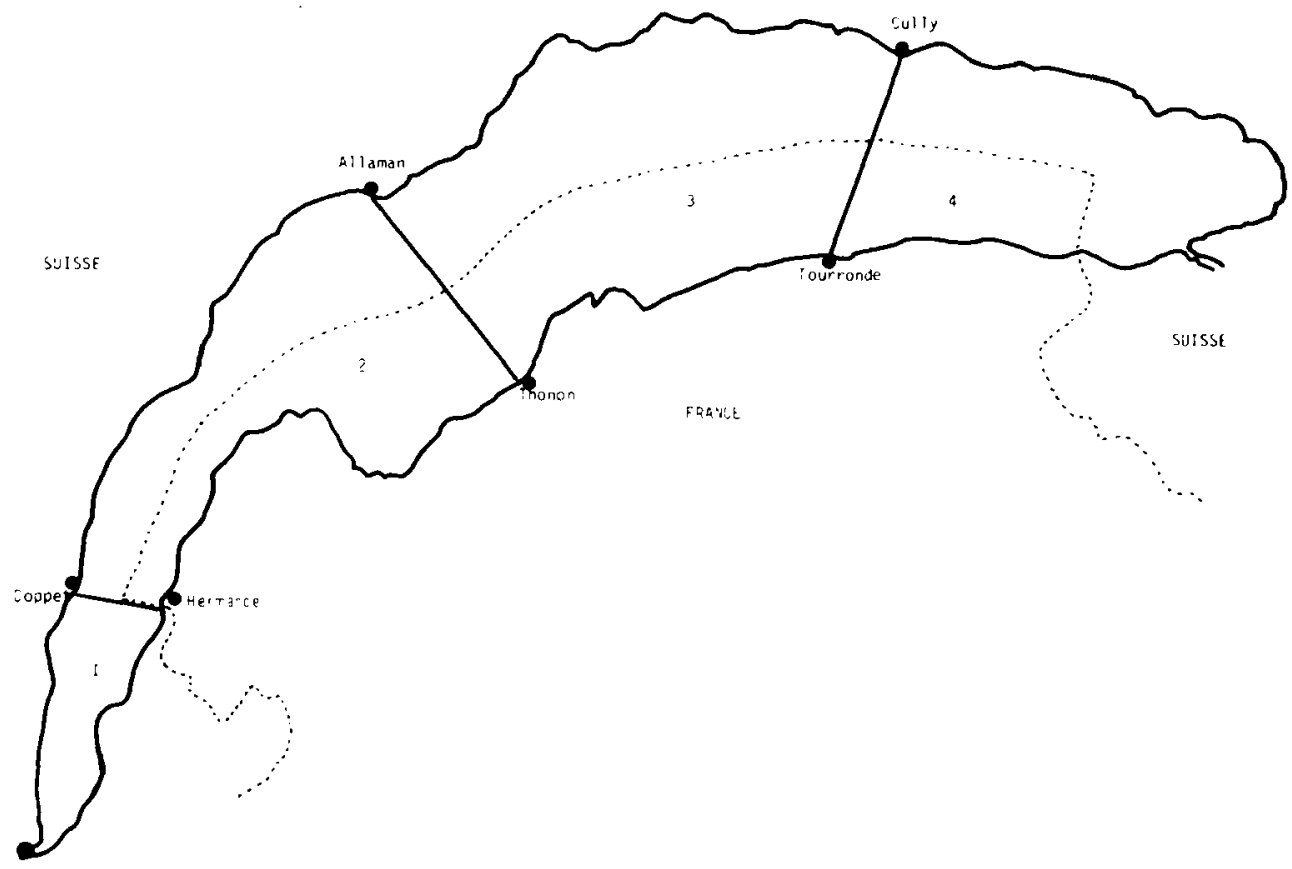

L'effort de pêche helvétique sur l'omble est peut être plus important qu'en France mais ceci ne saurait expliquer en totalité les meilleurs résultats notés en Suisse si les ombles n'avaient pas réellement tendance à migrer et à se tenir le long des rivages suisses.

L'adaptation d'ombles de pisciculture à la vie sauvage se fait-elle sans difficulté?. On ne dispose pas de données sur le régime alimentaire des poissons après leur lâcher dans le lac; toutefois, il a été possible de noter qu'un omble de 14 centimètres de longueur totale, ayant reçu une marque dorsale, s'était accoutumé 101 jours après son déversement à se nourrir de cladocères planctoniques (Daphnies et Leptodora). Ce 
poisson avait gagné 24 grammes et s'était allongé de 4 centimètres; une telle croissance est comparable à celle qui a été évaluée plus haut et on peut supposer que les jeunes ombles de pisciculture s'habituent sans trop de peine à trouver dans le lac la nourriture qui est celle de leurs congénères sauvages (2).

Le très net décalage de croissance entre les ombles de pisciculture et ceux lâchés au lac à l'âge de deux hivers révèle sans doute l'existence d'un problème trophique. L'omble adulte se nourrit le plus souvent aux dépens du zoobenthos profond $(2-4)$ et il semble ne plus trouver dans les lieux qu'il fréquente les proies qui lui seraient nécessaires.

\section{Comportement des ombles hybrides marqués.}

1095 hybrides de Saumon de Fontaine Salvelinus fontinalis Mich. et d'omble chevalier ont été marqués et lâchés dans le Léman en 1961 et en 1966

Les résultats des déversements ont été décevants : il n'a été repris que $2,55 \%$ de l'effectif lâché initialement et le tiers de ces poissons a été pêché dans des affluents du lac. En moins d'un an il n'a plus été revu aucun de ces hybrides qui paraissent donc ne pas être intéressants à propager, du moins dans le Léman.

\section{Les ombles de pisciculture et la pêche dans le Léman.}

Les pêcheurs amateurs ne bénéficient que de $13,75 \%$ des reprises d'ombles marqués, dont le reste, c'est-à-dire la majeure partie, profite aux pêcheurs professionnels. D'autre part, il a été déjà vu plus haut que les captures les plus nombreuses semblent se produire en Suisse $(61,7 \%$ contre $38,3 \%$ en France).

Pour autant que les déclarations des pêcheurs soient exactes, il apparaît que le filet à perches est l'engin qui capture le plus fréquemment des ombles marqués (Tableau 6). Si cette constatation s'applique également aux ombles non marqués, la pêche professionnelle de l'omble se pratiquerait davantage le long des rives que dans les zones profondes.

Tableau 6 : Nature des engins avec lesquels ont été capturés les ombles marqués. Résultats exprimés en pourcentage du nombre total de réponses fournies par les pêcheurs.

\begin{tabular}{|c|c|c|c|c|c|}
\hline $\begin{array}{l}\text { absence de ren- } \\
\text { seignements sur } \\
\text { le mode de capture }\end{array}$ & $\begin{array}{c}\text { Renseignements } \\
\text { imprecis }\end{array}$ & $\begin{array}{l}\text { rilets } \\
\text { Derches }\end{array}$ & Pics & $\begin{array}{l}\text { Filets d } \\
\text { ombies }\end{array}$ & $\begin{array}{c}\text { Tramails a } \\
\text { lottes }\end{array}$ \\
\hline $0:$ & $21.73 \quad 8$ & $32.69 \div$ & $11.73 \%$ & $5.65 \div$ & $2.20 \%$ \\
\hline
\end{tabular}

La période de plus grande capture d'ombles marqués par les pêcheurs professionnels se situe en juillet (Tableau 7); les mois de mars, juin et octobre semblent également favorables.

Tableau 7 : Pourcentage des captures d'ombles marqués selon les mois de I'année pour les pêcheurs professionnels et amateurs.

\begin{tabular}{|c|c|c|c|c|c|c|c|c|c|c|c|c|}
\hline 1tois & 21 & 92 & 33 & 94 & 35 & 35 & $\Rightarrow$ & 09 & 39 & 20 & 11 & 22 \\
\hline $\begin{array}{l}\text { Pécneurs pro- } \\
\text { fessionne?'s }\end{array}$ & 3.46 & $\therefore \therefore$ & $1 ? .74$ & 3.3: & 5.99 & 12.74 & 20.09 & 3.93 & 7.35 & $: 0.23$ & 2.95 & $4.4 z$ \\
\hline $\begin{array}{l}\text { Pëcheurs } \\
\text { amateurs }\end{array}$ & 2 & $\therefore .55$ & $5 . \equiv 6$ & $=$ & 2 & $1 .: 2$ & :2.11 & 5.56 & 44.44 & 16.66 & 0 & 0 \\
\hline
\end{tabular}

Pour les pêcheurs amateurs, c'est le mois de septembre qui est le meilleur, prolongé par le mois d'octobre, avant la fermeture de la pêche.

Le nombre de poissons atteignant la taille légale de capture détermine le succès d'un déversement. Ce succès est loin d'être éclatant pour les ombles marqués déversés à la longueur moyenne totale de 13 centimètres dont seulement $0,77 \%$ atteignent la taille légale de capture de 26 centimètres. Des ombles de deux hivers et de 23,8 centimètres de longueur totale moyenne fournissent des résultats moins mauvais puisque $2,62 \%$ d'entre eux atteignent ou dépassent la taille légale. D'ailleurs ces mêmes ombles contribuent à la reproduction du stock indigène puisque trois d'entre eux ont été jusqu'à 
présent retrouvés sur les frayères et que ce chiffre sera probablement dépassé quand le temps d'expérience aura été un peu plus long.

De l'ensemble de ces constatations relatives au marquage, il est possible de tirer quelques conclusions pratiques sur le repeuplement du Léman avec des ombles élevés en pisciculture.

- Le repeuplement avec des ombles de deux hivers d'une taille supérieure à 20 centimètres semble donner de meilleurs résultats que ceux qu'on peut enregistrer avec des ombles d'un hiver mesurant 13 centimètres. Lorsqu'il sera possible de marquer convenablement des estivaux ou des alevins, il faudra apprécier leur efficacité dans les repeuplements.

- Tous les déversements d'ombles semblent profiter davantage aux côtes suisses qu'aux côtes françaises. Les immersions dans la zone située entre la Dranse et Meillerie devraient toutefois être un peu plus avantageuses pour les pêcheurs français.

- Pour des ombles de 13 centimètres de longueur totale, les déversements effectués en mai, juin et juillet conduisent aux meilleurs retours de marques.

- La croissance des ombles adultes du Léman paraît retardée par rapport à celle des ombles de pisciculture du même àge. L'ornble du Léman semble ne plus trouver dans le lac des conditions trophiques aussi propices que celles de jadis. La désoxygénation des fonds, la présence de corps indésirables (mercure, dérivés organiques halogénés) et l'appauvrissement biologique qui en résulte sans doute, pourraient être tenus pour responsables. La descendance des ombles eux-mêmes pourrait être affectée par les fortes teneurs des œufs en PCB (5).

- L'omble, prédateur benthique, de grande valeur commerciale, est sûrement menacé dans le Léman et toutes les mesures permettant de le protéger ou d'aider à l'accroissement de son stock devraient être tentées avant qu'il n'ait totalement disparu.

\section{Références bibliographiques}

(1) DUMAS J., 1978. Premières observations de cryomarquages sur les adultes de saumon atlantique (Salmo salar L.) de la Nivelle marqués au stade smolt. Bulf. fr. Pisc., $270: 218-222$.

(2) FROST W.E., 1977. The food of char, Salvelinus willughbi (Günther) in Windermere J. Fish. Biol., $11: 531-547$.

(3) HOGLUND J. et ASTRAND M., 1973. Preferences among juvenile char, Salvelinus alpinus, to intraspecific odors and water currents studied with the fluvarium technique. Inst. Freswater Res. Drottningholm Rep., 53: 21-30.

(4) JOHNSON L., 1980. The arctic charr, Salvelinus alpinus, in Charrs salmonid fishes of the Genus Salvelinus. Edited by Balon E.K. Dr W. Junk bv Publishers P.O.Box 137132501 ES La Haye, Pays-Bas : 15-98.

(5) MONOD G., 1981. Communication personnelle.

(6) RUHLÉ Ch., 1977. Biologie und Bewirtschaftung des Seesaiblings (Salvelinus alpinus L.) in Zugersee. Schweiz. Z. Hydrol., 39, $1: 12-45$ 